\title{
Discovery of a 12 billion solar mass black hole at redshift 6.3 and its challenge to the black hole/galaxy coevolution at cosmic dawn
}

\author{
Xue-Bing Wu ${ }^{1,2}$, Feige Wang ${ }^{1}$, Xiaohui Fan ${ }^{3,2}$, Weimin $\mathbf{Y i}^{4}$, Wenwen \\ Zuo $^{5}$, Fuyan Bian ${ }^{6}$, Linhua Jiang ${ }^{2}$, Ian D. McGreer ${ }^{3}$, Ran Wang ${ }^{2}$, \\ Jinyi Yang ${ }^{1}$, Qian Yang ${ }^{1}$, David Thompson ${ }^{3}$ and Yuri Beletsky ${ }^{7}$ \\ ${ }^{1}$ Department of Astronomy, School of Physics, Peking University, Beijing 100871, China \\ email: wuxb@pku.edu.cn \\ ${ }^{2}$ Kavli Institute for Astronomy and Astrophysics, Peking University, Beijing 100871, China \\ ${ }^{3}$ Steward Observatory, University of Arizona, Tucson, AZ, United States \\ ${ }^{4}$ Yunnan Astronomical Observatories, Chinese Academy of Sciences, Kunming, China \\ ${ }^{5}$ Shanghai Astronomical Observatory, Chinese Academy of Sciences, Shanghai, China \\ ${ }^{6}$ Mount Stromlo Observatory, Australia National University, Weston Creek, ACT, Australia \\ ${ }^{7}$ Las Campanas Observatory, Carnegie Institution of Washington, La Serena, Chile
}

\begin{abstract}
The existence of black holes with masses of about one billion solar masses in quasars at redshifts $z>6$ presents significant challenges to theories of the formation and growth of black holes and the black hole/galaxy co-evolution in the early Universe. Here we report a recent discovery of an ultra-luminous quasar at redshift $z=6.30$, which has an observed optical and near-infrared luminosity a few times greater than those of previously known $z>6$ quasars. With near-infrared spectroscopy, we obtain a black hole mass of about 12 billion solar masses, which is well consistent with the mass derived by assuming an Eddington-limited accretion. This ultra-luminous quasar with at $z>6$ provides a unique laboratory to the study of the mass assembly and galaxy formation around the most massive black holes at cosmic dawn. It raises further challenges to the black hole/galaxy co-evolution in the epoch of cosmic reionization because the black hole needs to grow much faster than the host galaxy.
\end{abstract}

Keywords. black hole physics, galaxies: active, galaxies: nuclei, galaxies: evolution, quasars: general, quasars: emission lines, early universe

\section{Introduction}

So far, about 40 quasars at redshifts $z>6$ have been discovered (Fan et al. 2003, Jiang et al. 2007, Willott et al. 2007, Jiang et al. 2008, Willott et al. 2010a, Mortlock et al. 2011, Venemans et al. 2013, Banados et al. 2014). Each contains a black hole with a mass of about one billion solar masses $\left(10^{9} M_{\odot}\right)$ (Jiang et al. 2007, Mortlock et al. 2011, Venemans et al. 2013, Willott et al. 2003, Kurk et al. 2007, Willott et al. 2010b, De Rosa et al. 2011, De Rosa et al. 2014). The existence of such black holes when the Universe was less than 1 billion years old presents substantial challenges to theories of the formation and growth of black holes and the coevolution of black holes and galaxies at cosmic dawn(Volonteri 2012).

High redshift quasars have been efficiently selected using a combination of optical and near-infrared colours(Willott et al. 2007, Jiang et al. 2008). We have carried out a systematic survey of quasars at $z>5$ using photometry from the Sloan Digital Sky Survey (SDSS)(York et al. 2000), the two Micron All Sky Survey (2MASS)(Skrutskie 
et al. 2006) and the Wide-field Infrared Survey Explorer (WISE)(Wright et al. 2010), resulting in the discovery of a significant population of luminous high redshift quasars (Wang et al. 2015, Yi et al. 2014).

One of these new quasars, SDSS J010013.02 + 280225.8 (hereafter J0100 + 2802), is an ultra-luminous quasar at redshift $z=6.30$. It has an optical and near-infrared luminosity a few times greater than those of previously known $z>6$ quasars, and has a black hole with a mass of $\sim 1.2 \times 10^{10} M_{\odot}$ (for details see $\mathrm{Wu}$ et al. (2015)). It is the most luminous quasar at redshift $z>6$ with the most massive black hole in the early Universe.

\section{Target selection and observations}

$\mathrm{J} 0100+2802$, was selected as a high-redshift quasar candidate with the highest priority in our program due to its red optical colour (with SDSS AB magnitudes $i_{A B}=20.84 \pm$ 0.06 and $\left.z_{A B}=18.33 \pm 0.03\right)$ and a photometric redshift of $z \simeq 6.3$. It has bright detections in the 2MASS $J, H$ and $K_{s}$ bands with Vega magnitudes of $17.00 \pm 0.20$, $15.98 \pm 0.19$, and $15.20 \pm 0.16$, respectively; it is also strongly detected in WISE, with Vega magnitudes in W1 to W4 bands of $14.45 \pm 0.03,13.63 \pm 0.03,11.71 \pm 0.21$, and $8.98 \pm 0.44$. Its colour in the two bluest WISE bands, W1-W2, clearly separates it from the bulk of stars in our Galaxy (Wu et al. 2012). It is undetected in both radio and X-ray bands by the wide-area, shallow survey instruments.

Initial optical spectroscopy on J0100 + 2802 was carried out on 29 December, 2013 with the Lijiang 2.4-m telescope in Yunnan, China. The low resolution spectrum clearly shows a sharp break at about $8,800 \AA$ A consistent with a quasar at a redshift beyond 6.2. Two subsequent optical spectroscopic observations were conducted on 9 and 24 January 2014 respectively with the 6.5-m Multiple Mirror Telescope (MMT) and the twin 8.4-m mirror Large Binocular Telescope (LBT) in the USA. The Ly $\alpha$ line shown in the spectra confirms that $\mathrm{J} 0100+2802$ is a quasar at redshift of $6.30 \pm 0.01$. The equivalent width of the $\operatorname{Ly} \alpha+\mathrm{N} \mathrm{V}$ emission lines as measured from the LBT spectrum is roughly $10 \AA$, suggesting that J0100 + 2802 is probably a weak-line quasar (WLQ) (Fan et al. 1999).

We use the multiwavelength photometry to estimate the optical luminosity at restframe $3,000 \AA\left(L_{3000}\right)$, which is consistent with that obtained from K-band spectroscopy (see below). The latter gives a more reliable value of $3.15 \pm 0.47 \times 10^{47} \mathrm{ergs} \mathrm{s}^{-1}$, adopting a $\Lambda$ CDM cosmology with $H_{0}=70 \mathrm{~km} \mathrm{~s}^{-1} M p c^{-1}, \Omega_{M}=0.30$ and $\Omega_{\Lambda}=0.7$. Assuming an empirical conversion factor from the luminosity at $3,000 \AA$ to the bolometric luminosity (Shen et al. 2011), this gives $L_{\mathrm{bol}}=5.15 \times L_{3,000}=1.62 \times 10^{48} \mathrm{ergs} \mathrm{s}^{-1}=4.29 \times 10^{14} L_{\odot}$ (where $L_{\odot}$ is the solar luminosity). The luminosity of this quasar is roughly 4 times greater than that of the luminous $z=6.42$ quasar SDSS J1148+5251 (Fan et al. 2003), and 7 times greater than that of the most distant known quasar ULAS J1120+0641 at $z=7.085$ (Mortlock et al. 2011).

The LBT spectrum of J0100 + 2802 (see Fig. 1) exhibits a deep Gunn-Peterson absorption trough (Gunn \& Peterson 1965) blueward of the Ly $\alpha$ emission. Complete GunnPeterson absorption can also be seen in the $\operatorname{Ly} \alpha, \operatorname{Ly} \beta$ and $\operatorname{Ly} \gamma$ transitions. Statistically significant transmission peaks are detected at $z=5.99$ in both the Ly $\alpha$ and Ly $\beta$ troughs, and an additional transmission peak is detected at $z=5.84$ in the Ly $\beta$ trough. The proximity zone size, defined as the point where the transmitted flux first drops by a significant amount to below $10 \%$ (ignore small absorption leaks) of the quasar extrapolated continuum emission after the spectrum is smoothed to a resolution of $20 \AA$ (Fan et al. 2006), is much larger for J0100 $+2802(7.9 \pm 0.8 \mathrm{Mpc} ; 1 \mathrm{Mpc}$ is about 3.26 million light years) than that of other SDSS quasars at $z>6.1$ (Fan et al. 2006, Carilli et al. 2010). Its large 


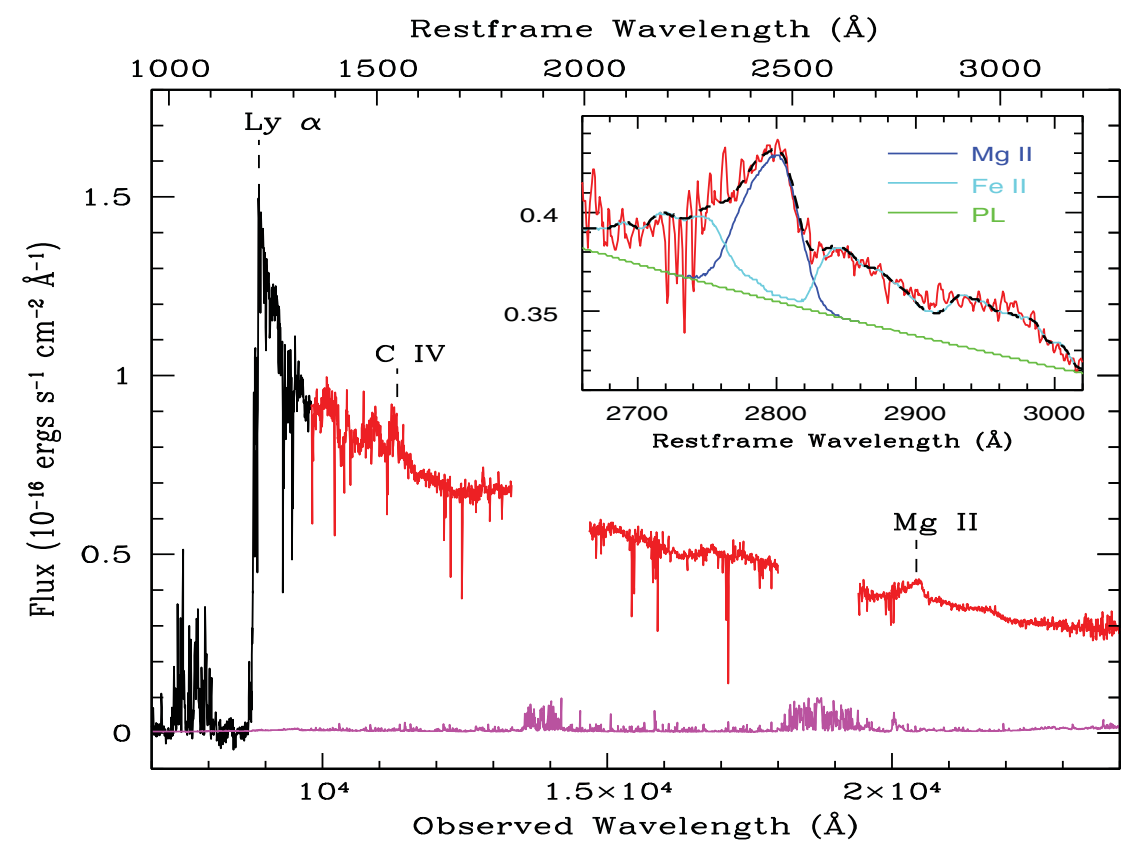

Figure 1. The optical/near-infrared spectrum of J0100 +2802 . The black line shows the LBT optical spectrum and the red line shows the combined Magellan and Gemini near-infrared J,H,K-band spectra. The magenta line shows the noise spectrum. In the inset, the green, cyan, and blue solid lines show the power law (PL), Fe II and Mg II fitting components. The black dashed line shows the sum of these components in comparison with the observed spectrum.

proximity zone size is expected from the higher level of photo-ionization dominated by quasar radiation.

We obtained the near-infrared J,H,K-band spectra with Gemini and Magellan telescopes on 6 August and 7 October 2014, respectively. Fig. 1 shows the combined optical/nearinfrared spectrum of $\mathrm{J} 0100+2802$ and the result of fitting the $\mathrm{Mg}$ II emission line. The Mg II Full Width at Half Maximum (FWHM) is $5,130 \pm 150 \mathrm{~km} \mathrm{~s}^{-1}$, and the continuum luminosity at the rest-frame wavelength of $3,000 \AA$ is $3.15 \pm 0.47 \times 10^{47} \mathrm{ergs} \mathrm{s}^{-1}$. After applying a virial black hole mass estimator based on the Mg II line (McLure \& Dunlop $2004)$, we estimate its black hole mass to be $(1.24 \pm 0.19) \times 10^{10} M_{\odot}\left(M_{\odot}\right.$. Assuming that this quasar is accreting at Eddington accretion rate and the bolometric luminosity is close to the Eddington Luminosity $\left(L_{E d d}=1.3 \times 10^{38}\left(M / M_{\odot}\right)\right)$, similar to other $z>6$ quasars (Willott et al. 2010b), leads to a black hole mass of $1.3 \times 10^{10} M_{\odot}$ for $\mathrm{J} 0100+2802$. Therefore, our observations strongly indicate that J0100 +2802 harbors a black hole of mass about $1.2 \times 10^{10} M_{\odot}$, the first such system known at $z>6$.

\section{Discussions}

Although gravitational lensing is a possible explanation for its high luminosity, we do not expect a large lensing magnification for $\mathrm{J} 0100+2802$. An LBT K-band image with seeing of $0.4^{\prime \prime}$ shows a morphology fully consistent with a single point source; and the large quasar proximity zone size further supports a high UV luminosity consistent with the expected photoionization scaling (Haiman 2005). However, absorption features at different redshift have been identified from its near-infrared spectroscopy, implying the existence of abundant intervening materials along the line of sight (Wu et al. (2015)). 
$\mathrm{J} 0100+2802$ is the only quasar with a bolometric luminosity higher than $10^{48}$ ergs $s^{-1}$ and a black hole mass larger than $5 \times 10^{9} M_{\odot}$ at $z \geqslant 6$. Its discovery in the entire SDSS footprint $\left(\sim 13,000 \mathrm{deg}^{2}\right)$ is broadly consistent with the extrapolation of SDSS $z \simeq 6$ quasar luminosity function (Fan et al. 2006). The number density of such objects would set strong constraints on the early growth of supermassive black hole and the evolution of high-redshift quasar black-hole mass function (Willott et al. 2010a, Willott et al. 2010b). $\mathrm{J} 0100+2802$ with a $1.2 \times 10^{10} M_{\odot}$ black hole at $z=6.30$ presents significant challenge to the Eddington-limited growth of black holes in the early Universe (Willott et al. 2010b, Volonteri 2012). Its existence also strengthens the claim that supermassive black holes in the early Universe probably grew much more quickly than their host galaxies, as argued from a molecular gas study of $z \simeq 6$ quasars (Wang et al. 2010), Therefore, as the most luminous quasar known to date at $z>6$, J0100 + 2802 will be a unique resource for the future study of the mass assembly and galaxy formation around the most massive black holes at cosmic down (Fan et al. 2006).

\section{Acknowledgement}

This work is supported by NSFC (11033001, 11373008, 11443002 \& 11533001), NSF (AST 08-06861 \& 11-07682), the Strategic Priority Research Program of the CAS (XDB09000000), and the National Key Basic Research Program of China (2014CB845700). We acknowledge the use of SDSS, 2MASS, WISE data and the Lijiang 2.4-m, MMT, LBT, Gemini and Magellan telescopes.

\section{References}

Banados, E. et al. 2014, AJ, 148, 14

Carilli, C. L. et al. 2010, ApJ, 714, 834

De Rosa, G. et al. 2011, ApJ, 739, 56

De Rosa, G., et al. 2014, ApJ, 790, 145

Fan, X., et al. 1999, ApJ (Letters), 526, L57

Fan, X. et al. 2003, AJ, 125, 1649

Fan, X., Carilli, C. L., \& Keating, B. 2006, ARAA, 44, 415

Gunn, J. E. \& Peterson, B. A. 1965, ApJ, 142, 1633

Haiman, Z. et al. 2005, AJ, 623, 627

Jiang, L. et al. 2007, AJ, 134, 1150

Jiang, L., et al. 2008, AJ, 135, 1057

Kurk, J. D. et al. 2007, ApJ, 669, 32

McLure, R. J. \& Dunlop, J. S. 2004, MNRAS, 352, 1390

Mortlock, D. J. et al. 2011, Nature, 474, 616

Shen, Y. et al. 2011, ApJS, 194, 45

Skrutskie, M. F. et al. 2006, AJ, 131, 1163

Venemans, E. P. et al. 2013, ApJ, 779, 24

Volonteri, M. 2012, Science, 337, 544

Wang, F., et al. 2015, ApJ (Letters), 807, L9

Wang, R., et al. 2010, ApJ, 714, 699

Willott, C. J., et al. 2007, AJ, 134, 2435

Willott, C. J. et al. 2010, AJ, 139, 906

Willott, C. J. et al. 2010a, AJ, 140, 546

Willott, C. J., McLure, R. J., \& Jarvis, M. J. 2003, ApJ (Letters), 587, L15

Wright, E. L. et al. 2010, AJ, 140, 1868

Wu, X.-B. et al. 2012, AJ, 144, 49

Wu, X.-B., et al. 2015, Nature, 518, 512

Yi, W., et al. 2014, ApJ (Letters), 795, L29

York, D. G. et al. 2000, AJ, 120, 1579 